\title{
Estimation of the cosmic ray ionization in the Earth's atmosphere during GLE71
}

\section{L.I. Dorman ${ }^{1}$}

Israel Cosmic Ray \& Space Weather Centre and Emilio Ségre Observatory, affiliated to Tel Aviv University, Golan Research Institute, and Israel Space Agency, Tel Aviv, Israel

N.V. Pushkov's IZMIRAN Russian Academy of Science, Moscow, Russia

E-mail: lid@physics.technion.ac.il

\section{P. Paschalis}

Nuclear and Particle Physics Department, Faculty of Physics

National and Kapodistrian University of Athens

E-mail:ppaschalis@phys.uoa.gr

\section{Plainaki}

Institute of Space Astrophysics and Planetology, INAF

Via del Fosso del Cavaliere 100, 00133 Rome, Italy

E-mail: Christina.Plainaki@iaps.inaf.it

\section{H. Mavromichalaki}

Nuclear and Particle Physics Department, Faculty of Physics

National and Kapodistrian University of Athens

E-mail: emavromi@phys.uoa.gr

DYASTIMA is an application, based on Geant4, which simulates the cascades of particles that are generated due to the interactions of cosmic ray particles with the atmospheres of the planets. The first version of DYASTIMA has been successfully applied to the Earth's atmosphere, providing results that are in accordance with the publications of other models. Since then, important improvements and extensions have been made to the application, including a graphical user interface environment that allows the more effective management of the configuration parameters. Also, the actual modelling of the atmosphere has been changed allowing the definition of more complex cases and at the same time providing, in a more efficient way (with respect to the program's previous version) enhanced outputs. In this work, we combine the new version of DYASTIMA with the NMBANGLE PPOLA model, that estimates the spectrum of SEPs during relativistic proton events using ground level neutron monitor data from the worldwide network. Such a joint model has as a primary scope the simulation of a SEP event and of its secondary products at different altitudes in the Earth's atmosphere, providing at the same time estimation of the respective ionization rates and of their spatial and temporal dependence. We apply this joint model to GLE 71, on 17 May 2012, and we discuss the results.

The 34th International Cosmic Ray Conference

30 July- 6 August, 2015

The Hague, The Netherlands

${ }^{1}$ Speaker 


\section{Introduction}

Galactic cosmic rays and solar energetic particles that enter the atmosphere of a planet interact with the molecules of the atmosphere and produce cascades of particles. The study of these cascades is of great importance since they constitute the connection between the cosmic rays and their affection to the planet. Simulation techniques are effective computational tools to study these cascades. The CORSIKA [1] and the PLANETOCOSMICS [2] are two well known applications which are successfully used for this purpose. Moreover, CRII [3] is a model that calculates the cosmic rays induced ionization in the atmosphere of the Earth. Recently, a new simulation application which is based on Geant 4 and is called DYASTIMA, has been developed $[4,5]$. DYASTIMA has already been applied in the past for the study of cascades in Earth during quiet space weather conditions [6] and for the determination of ionization in the Venusian atmosphere [7]. In this work we use DYASTIMA in order to calculate the ionization of the Earth's atmosphere during GLE 71 on 17 May 2012. For this task DYASTIMA is combined with NMBANGLE PPOLA model [8], which estimates the spectrum of a SEP event by using the ground level neutron monitor data.

\section{The DYASTIMA model}

The study of the particle cascades via simulations is a complicated procedure due to the numerous input and output parameters that have to be considered. The most indicative input parameters that should be managed, combined and transformed in order to set up the Geant4 [4, 5] are the structure of the atmosphere, the magnetic field, the spectra of the incoming particles, the physical interactions that are considered, the energy cuts that are applied in order to accelerate the simulation and the altitudes in which the information about the cascade is registered. Moreover, a huge amount of information should be processed during the simulation, regarding the energy, the direction, the energy deposit, the arrival time and the position of each particle of the cascade. Apart from the accuracy, which is always the main goal of any computational technique, the challenging point about the simulation of cascades is the handling of the input and output parameters in an efficient way. This will allow the extended parameterization of the simulation and the collection of the necessary information about the cascade, with good time performance. DYASTIMA has been developed in consideration of the requirements mentioned above. The first version of DYASTIMA [6] makes use of text files for the configuration of the simulation. The new improved version allows more parameterization and introduces a Graphical User Interface (GUI), which increases the ease of use by non experienced users. The GUI checks the validity of the settings and generates all the necessary parameters for Geant4. The Geant 4 simulation is then automatically executed as a console application. A screen-shot of the GUI is shown in figure 1. 


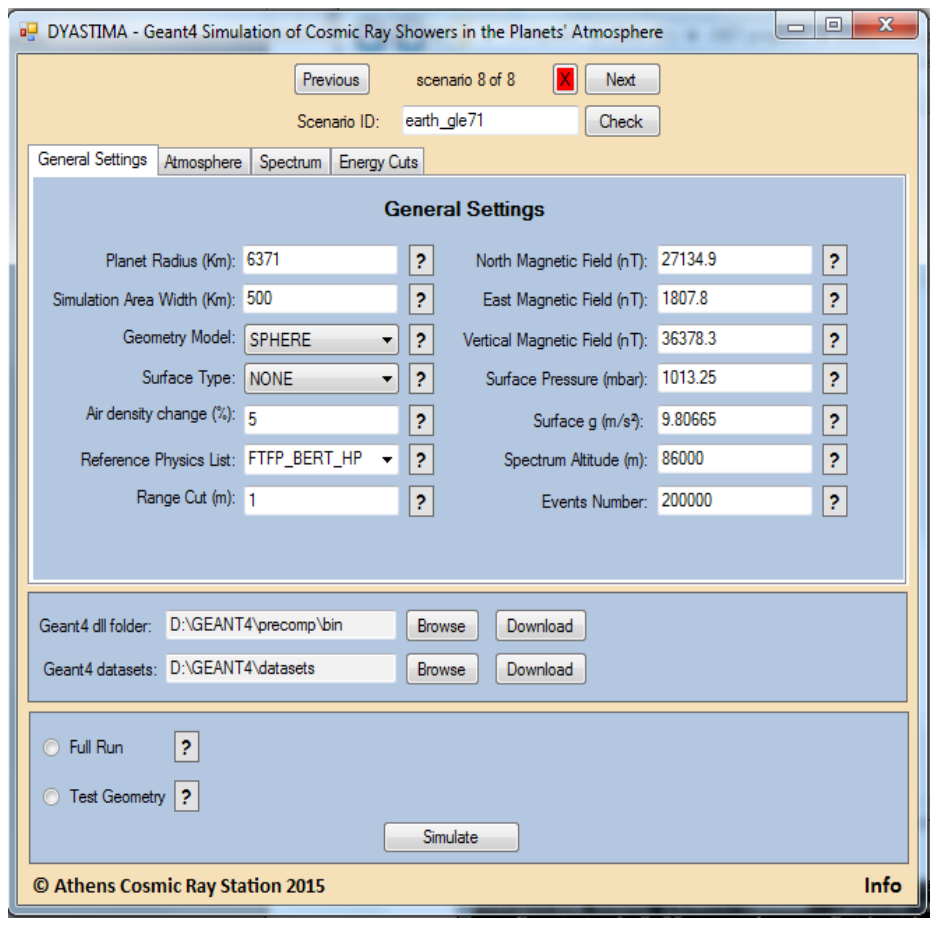

Figure 1: Graphical User Interface of DYASTIMA

From the operational point of view, the atmosphere of the planet is defined by its composition, its temperature profile and the surface pressure. Starting from the bottom of the atmosphere and by using the hydrostatic equation, DYASTIMA divides the atmosphere in slices each one of which has a constant composition and density. The division is performed in such a way that the density change of the sequential slices is smaller than a user defined percentage. The result of the division is used for the construction of the simulation area in Geant4. Two different models have been implemented in Geant4, regarding the geometry of the area. In the first model the slices are rectangular boxes, while in the second model the slices are spherical shells. The spherical model represents the curvature of the planet and provides better accuracy of the calculations with a trade-off in the time performance. Regarding the particles that enter the atmosphere, multiple types are allowed. For each particle type is required to define the differential spectrum by declaring energy - flux pairs as well as the solid angle of their incoming directions. According to the defined spectra and solid angles, DYASTIMA calculates the relative abundances of each particle type and sets a General Particle Source (GPS) in Geant4 that emits particles from the top of the atmosphere. For the physical interactions that take place during the simulation, DYASTIMA uses the reference physics lists of Geant 4 that are validated through the HEP experiments. A user defined magnetic field is applied to the simulation area in order to simulate the diversion of the particle tracks. Regarding the output, DYASTIMA registers the energy and the altitude of the new particles that are generated and the energy, the direction, the time and the energy deposit of the particles that cross predefined tracking layers. The registration is performed by using the Stacking Action and Stepping Action classes of Geant4. The output is written in .csv files after the simulation is finished. Since the number of particles that are generated in the cascade is huge, especially through the electromagnetic processes, DYASTIMA may set energy cuts for the production, simulation or tracking of 
particles. This accelerates the simulation with a trade-off to accuracy. A diagram that shows the operation of DYASTIMA is given in figure 2.

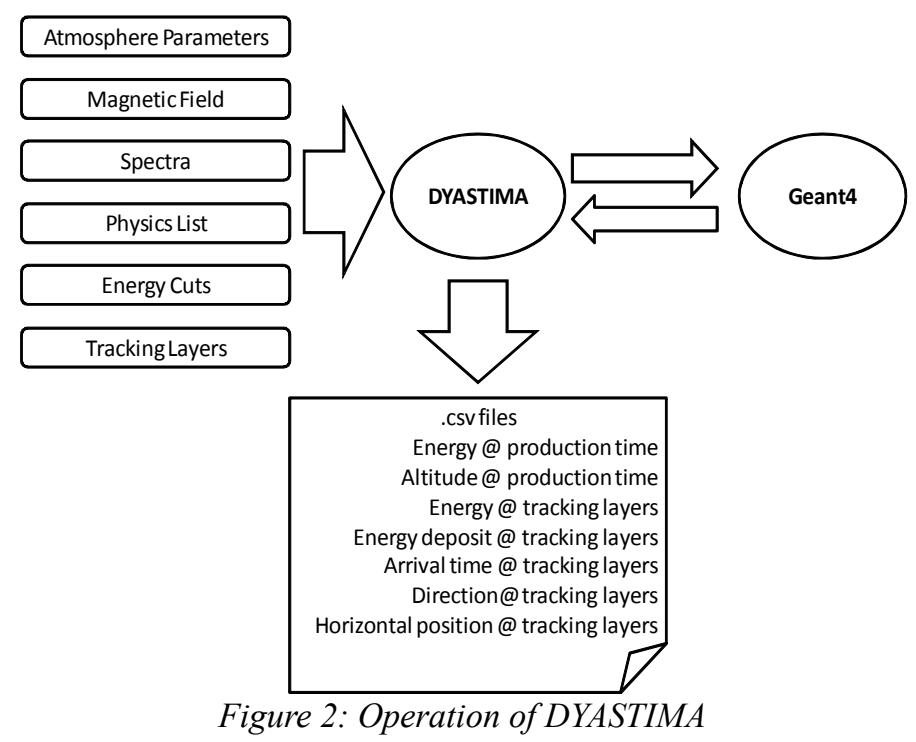

\section{The NMBANGLE PPOLA model}

The NMBANGLE PPOLA model ([8],[9]) is a modified version of the original NMBANGLE model [10], which is based on the coupling coefficient method [11, 12], applied numerous times in the past ([13], [14]). The NMBANGLE PPOLA model couples SEPs (primary particles) at a selected altitude in the Earth's atmosphere with the secondary ones detected by ground-level NMs during GLEs. This model dynamically calculates the SEP spectrum and the SEP flux spatial distribution at some altitude in the atmosphere. A power-law SEP spectrum with two free parameters (spectral index and amplitude) is assumed. SEPs are assumed to be protons in the model's current version. The details of the physics considered in the NMBANGLE and the NMBANGLE PPOLA models have been provided in the past ([8], [9], [10]). In this Section we only give a very brief description of the model.

The NMBANGLE PPOLA model uses as inputs the response of the worldwide NM network to the high-energy solar protons (i.e. $\geq 500 \mathrm{MeV}$ ) and the disturbance level of the geomagnetic field (through the use of the $\mathrm{k}_{\mathrm{p}}$ index). The NM network is treated as an integrated omnidirectional spectrometer able to measure the characteristics of the relativistic primary flux at a selected altitude $\left(\mathrm{h}_{0}=20 \mathrm{~km}\right)$ in the Earth's atmosphere. GLE data from NM stations widely distributed around the world are incorporated. In this context, the modeling of the NM response to an anisotropic SEP flux and the solving of the inverse problem can provide the actual characteristics of the relativistic SEPs that are responsible for the event. The results of the application of both NMBANGLE and NMBANGLE PPOLA models to past GLEs have been, in general, in good agreement with space observations, when available ([8], [9], [10]). We note that due to the particle motion inside the geomagnetic field, each ground level detector is capable of recording secondary particles produced by primaries arriving from a set of directions in space, depending on their energy, known as the station's asymptotic directions of viewing. For the 
evaluation of the NM directions of viewing, the method described by [15] is applied; the assumption that the Earth's magnetospheric field can be adequately described by the T89 model ([16]) is considered. In the particle trajectory calculation, the relativistic SEPs have been considered to have a vertical incidence at each neutron monitor location.

According to the NMBANGLE PPOLA model, the possible time variations, $\Delta \mathrm{N} / \mathrm{N}_{0}$, of the total neutron counting rate, $\mathrm{N}_{0}$, observed at cut-off rigidity $\mathrm{R}_{\mathrm{c}}$, geographic latitude $\varphi$ and longitude $\lambda$, at level $\mathrm{h}$ in the atmosphere and at some moment $\mathrm{t}$, are determined by the following expression:

$$
\frac{\Delta \mathrm{N}\left(R_{c}, h, t, t_{0}\right)}{\mathrm{N}_{0}\left(R_{c}, h, t_{0}\right)}=\int_{R_{c}}^{R_{u}} \frac{W\left(R, h, t_{0}\right) \cdot \Delta \mathrm{I}(R, \Omega(R, t), t)}{I_{o}\left(R, t_{0}\right)} d R=\int_{R_{c}}^{R_{u}} \frac{W\left(R, h, t_{0}\right) \cdot A(R, \Omega(R, t), t) \cdot b(t) \cdot R^{\gamma(t)}}{I_{o}\left(R, t_{0}\right)} d R
$$

where $\mathrm{W}\left(\mathrm{R}, \mathrm{h}, \mathrm{t}_{0}\right)$ is the rigidity-dependent coupling function (properly normalized to have values in the 0-1 range) between secondary and primary particles arriving at altitude $h_{0}$ (with $\left.h_{0}>h\right)$ in the Earth's atmosphere; $\Delta I(R, \Omega(R, t), t)=I(R, \Omega(R, t), t)-I_{0}\left(R, t_{0}\right)$ is the increase of the primary flux due to the arrival of SEPs at altitude $h_{o}$, assumed to follow a power law in rigidity, i.e. $\mathrm{I}(\mathrm{R},(\mathrm{R}, \mathrm{t}), \mathrm{t}) \mathrm{A}(\mathrm{R},(\mathrm{R}, \mathrm{t}), \mathrm{t}) \mathrm{b}(\mathrm{t}) \mathrm{R}^{\gamma(\mathrm{t})} ; \gamma(\mathrm{t})$ is the exponent of the power-law SEP spectrum; $A(R, \Omega, t)$ is a dimensionless normalized function that describes the spatially anisotropic SEP arrival at altitude $h_{o}$, with $\Omega(R, t)$ being the angle defined by the direction of viewing for vertical incidence at a NM for a rigidity $\mathrm{R}$ and the vertical direction of the apparent SEP source at altitude $\mathrm{h}_{0} ; \mathrm{R}_{\mathrm{u}}$ is the theoretical upper limit for the rigidity of the SEP particles, a parameter that in the model is specified a priori (here $8 \mathrm{GV}$ ) without a big loss in the estimation accuracy of the other parameters; $b(t)$ is the amplitude of the SEP differential flux (in protons $\mathrm{m}-2 \mathrm{~s}^{-1} \mathrm{sr}^{-1} \mathrm{GV}^{-1}$ ); and $\mathrm{I}_{0}\left(\mathrm{R}, \mathrm{t}_{0}\right)$ is the GCR differential flux (in protons $\mathrm{m}^{-2} \mathrm{~s}^{-1} \mathrm{sr}^{-1} \mathrm{GV}^{-1}$ ). In our model, we define $\mathrm{A}(\mathrm{R}, \Omega(\mathrm{R}, \mathrm{t}), \mathrm{t})$ as :

$$
\mathrm{A}(R, \Omega, t)=\exp \left(-n_{\alpha}(t)^{2} \sin ^{2} \frac{\Omega(R, t)}{2}\right)
$$

where $\mathrm{n}_{\alpha}(\mathrm{t})$ is a dimensionless parameter that characterizes the width of a primary solar particle beam, arriving at altitude $h_{o}$, around a specific location, whose latitude and longitude are free parameters for the model. Through the selection of the mathematical form described in Equation (2), the NMBANGLE PPOLA model parameterizes the level of the primary particle flux anisotropy by the use of variable $n_{\alpha}(t)$, which is dynamically determined after each model run. Big values for $n_{\alpha}(t)$ mean that the arriving SEP flux is narrowly distributed around a specific location at altitude $h_{0}$, whereas smaller values for $n \alpha(t)$ mean that the SEP flux is more widely distributed in longitudes and latitudes. This parameter is determined independently from the magnitude of the primary SEP intensity. However, it is the product of the anisotropy function with the primary spectrum that gives the total behavior of the primary flux responsible for the event registered at ground level. A least-square fitting technique based on the Levenberg Marquardt algorithm allows an efficient derivation of the optimal solution for each time interval and the determination of the GLE parameters evolution. Five-minute GLE data from NM 
stations of the worldwide network are incorporated to fit Equation (1). Each time represents the start of a five minute integrated time interval.

\section{Results and Discussion}

For this work, the International Standard Atmosphere [17] has been configured in DYASTIMA. The temprature and the density profile of the atmosphere that is used are shown in figure 3.

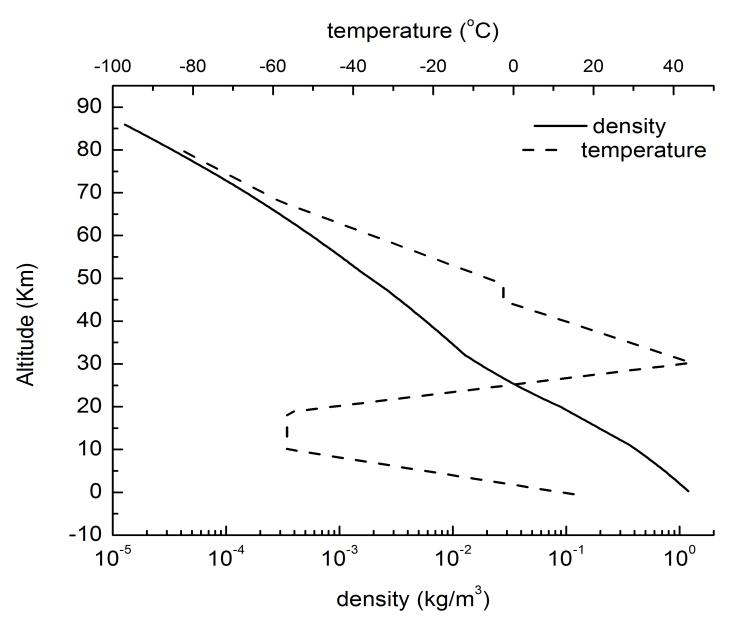

Figure 3: Temperature and density profile of the International Standard Atmosphere

The density change between the slices, in which the atmosphere is divided, is set to $5 \%$, resulting to a division of 224 slices. The calculation of the ionization rate has been performed for a location with $0 \mathrm{GV}$ cutoff rigidity by using the spectrum of protons during the main phase of GLE71, which is exported from the NMBANGLE PPOLA model. For comparison reasons, the calculations have also been performed by using the spectra during the solar minimum and solar maximum conditions. The corresponding spectra are extracted from the CREME model [18], which calculates the spectra at $1 \mathrm{AU}$. The exact spectra that were used were taken from [13] in which a power law tail was fitted in the spectra of CREME in order to extrapolate them up to $1 \mathrm{TeV} / \mathrm{n}$ (Fig. 4).
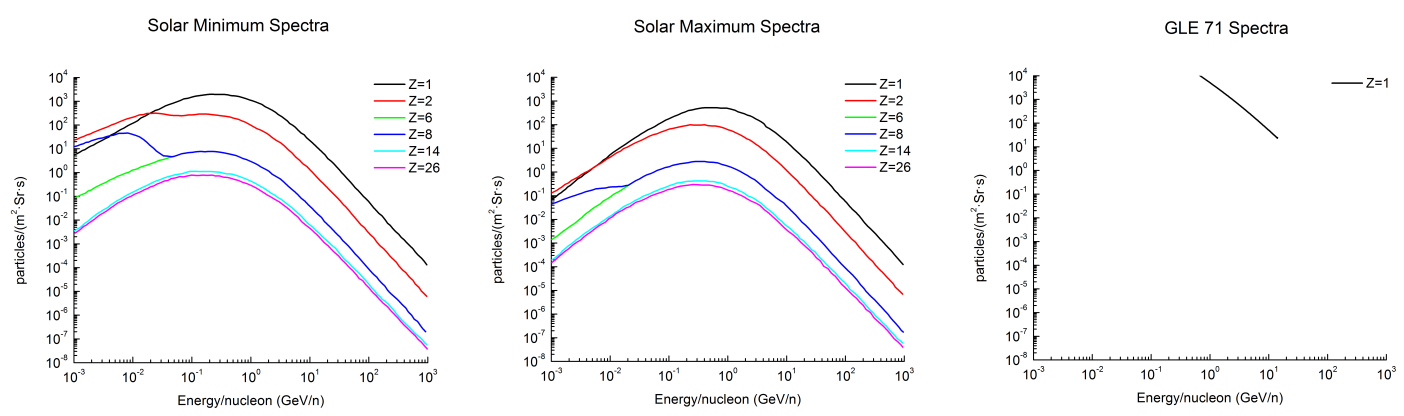

Figure 4: Spectra corresponding to solar minimum, solar maximum and main phase of GLE 71

The reference physics list that was used is the FTFP_BERT_HP recommended by Geant 4 for HEP and that uses a high precision model for the low energy neutrons. The calculation of the ionization rate was performed by simulating 300.000 particles entering the atmosphere (Fig. 5). 
The ionization is calculated in pairs $/ \mathrm{cm}^{3} \cdot \mathrm{s}$ and pairs $/ \mathrm{g} \cdot \mathrm{s}$ which is the unit used by CRII model [3]. For determing the number of produced pairs, we measure the ionization energy deposit at each tracking layer and divide the result by the average energy needed to produce one ion pair, that is $35 \mathrm{eV}[3,20]$.
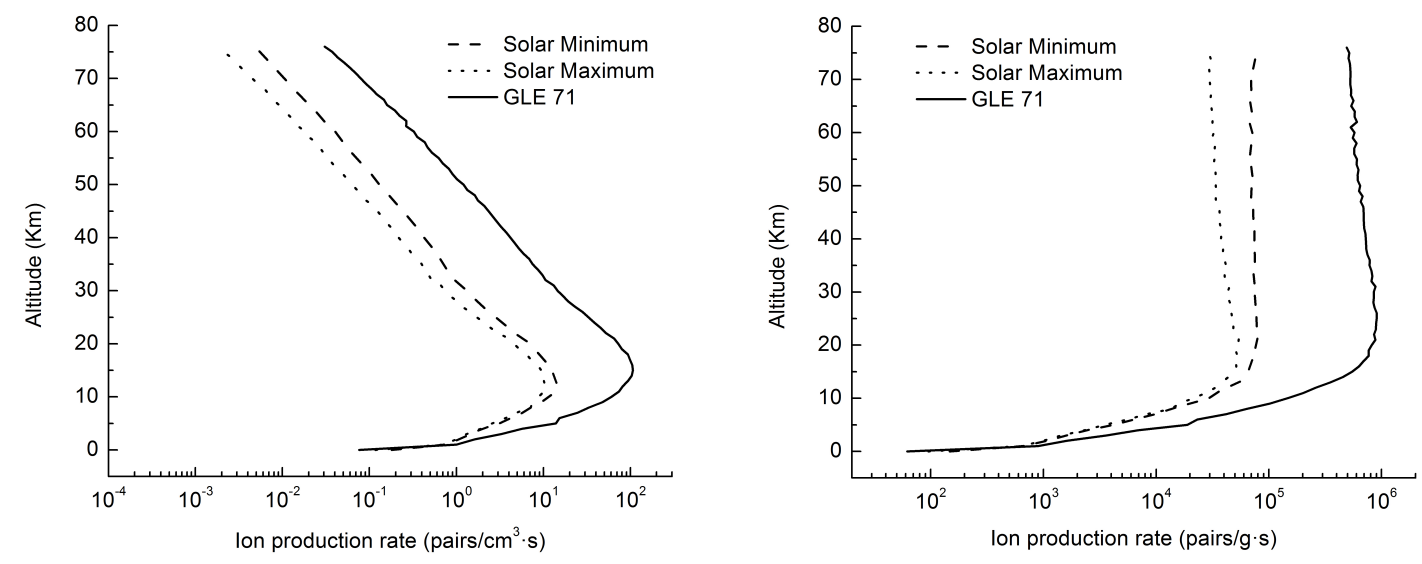

Figure 5: Ionization of the atmosphere in pairs $/ \mathrm{cm}^{3} \cdot \mathrm{s}$ and pairs $/ \mathrm{g} \cdot \mathrm{s}$ units

\section{Conclusion}

As it is expected, the ionization at solar maximum conditions is smaller than the ionization at solar minimum conditions, since the flow of the cosmic rays is smaller. During GLE 71 the ionization is significantly greater due to the increased flow of protons. Reffering to the pairs $/ \mathrm{cm}^{3} \cdot \mathrm{s}$ units, the maximum ionization at solar maximum is 10.4 pairs $/ \mathrm{cm}^{3} \cdot \mathrm{s}$ at $12 \mathrm{Km}$ altitude, at solar minimum is 14.6 pairs $/ \mathrm{cm}^{3} \cdot \mathrm{s}$ at $13 \mathrm{Km}$ altitude and at GLE 71 is 106.5 pairs $/ \mathrm{cm}^{3} \cdot \mathrm{s}$ at $15 \mathrm{Km}$ altitude. Reffering to the pairs $/ \mathrm{g} \cdot \mathrm{s}$ units, the maximum ionization at solar maximum is $54375 \mathrm{pairs} / \mathrm{g} \cdot \mathrm{s}$ at $21 \mathrm{Km}$ altitude, at solar minimum is $80250 \mathrm{pairs} / \mathrm{g} \cdot \mathrm{s}$ at $21 \mathrm{Km}$ altitude and at GLE71 is 905000 pairs/g·s at $26 \mathrm{Km}$ altitude. It is noticed that when using the pairs $/ \mathrm{g} \cdot \mathrm{s}$ units, the ionization rate is significant even at the top layers of the atmosphere due to the low density.

Acknowledgments: Many thanks to the colleagues of the NM stations for kindly providing the cosmic ray data used in this study in the frame of the High resolution Neutron Monitor database NMDB, funded under the European Union's FP7 Program (contract no. 213007).

\section{References}

[1] D. Heck, J. Knapp, J.N. Capdevielle, G. Schatz, T. Thouw, CORSIKA: A Monte Carlo Code to Simulate Extensive Air Showers, Forschungszentrum Karlsruhe GmbH, Karlsruhe (Germany)., Feb 1998, V + 90 p., TIB Hannover, D-30167 Hannover (Germany)

[2] L. Desorgher, E.O. Flückiger, M. Gurtner, The PLANETOCOSMICS Geant4 application, 36th COSPAR Scientific Assembly, Meeting abstract from the CDROM, \#2361, 2006

[3] I.G. Usoskin, G.A. Kovaltsov, Cosmic ray induced ionization in the atmosphere: Full modeling and practical applications, J. Geophys. Res., 111, D21206, 2006 
[4] S. Agostinelli, J. Allison, K. Amako, J. Apostolakis et al. [Geant4 Collaboration], Geant4 - $a$ simulation toolkit, NIM A, 506, 250-303, 2003

[5] J. Allison, K. Amako, J. Apostolakis, H. Araujo et al. [Geant4 Collaboration], Geant4 developments and applications, IEEE Transactions on Nuclear Science, 53, 270-278, 2006

[6] P. Paschalis, H. Mavromichalaki, L.I. Dorman, C. Plainaki, D. Tsirigkas, Application for the cosmic rays simulation of atmospheric showers using Geant4, New Astronomy, 33, 26-37, 2014

[7] C. Plainaki, P. Paschalis, D. Grassi, H. Mavromichalaki, M. Andriopoulou, Cosmic ray interactions with the Venusian atmosphere, EGU2015-3945

[8] C. Plainaki, H. Mavromichalaki, A. Belov, E. Eroshenko, M. Andriopoulou, V. Yanke, A new version of the NMBANGLE Model applied to the GLE60, Solar Physics,264, 339-254, 2010

[9] C. Plainaki, H. Mavromichalaki, M. Laurenza, M. Gerontidou, A. Kanellakopoulos, M. Storini, The Ground Level Enhancement of 2012 May 17: Derivation of solar proton event properties through the application of the NMBANGLE PPOLA model, The Astrophysical journal, 785:160, 2014

[10] C. Plainaki, A. Belov, E. Eroshenko, H. Mavromichalaki, V. Yanke, Modelling ground level enhancements: The event of 20 January 2005, J. of Geo. Res.-Space Physics, 112, A04102, 2007

[11] L.I. Dorman, Cosmic Ray Variations, Gostekhteorizdat, Moscow, 1957 (in English: USA Department of Defense, Ohio, 1958)

[12] L.I. Dorman, Cosmic Rays in the Earth's Atmosphere and Underground, Kluwer Academic Publishers, Dordreht/London/New York, 2004

[13] A. Belov, E. Eroshenko, H. Mavromichalaki, C. Plainaki, V. Yanke, Solar cosmic rays during the extremely high ground level enhancement on February 23, 1956, Annales Geophysicae, 224, 22812291,2005

[14] C. Plainaki, H. Mavromichalaki, A. Belov, E. Eroshenko, V. Yanke, Modeling the solar cosmic ray event of 13 December 2006 using ground level neutron monitor data, Adv. in Space Res., 43, 474479, 2009

[15] C. Plainaki, H. Mavromichalaki, A. Belov, E. Eroshenko, V. Yanke, Neutron Monitor asymptotic directions of viewing during the event of 13 December 2006, Adv. in Space Res., 43, 518-522, 2009

[16] N. A. Tsyganenko, A Magnetospheric Magnetic Field Model with a Warped Tail Current Sheet, Planet. Space Sci. 37, 5-20, 1989

[17] International Civil Aviation Organization, Manual of the ICAO Standard Atmosphere (extended to 80 kilometres (262 500 feet)), Doc 7488-CD, Third Edition, 1993

[18] A.J. Tylka et al., CREME96: a revision of the Cosmic Ray Effects on Micro-Electronics code, IEEE Transactions on Nuclear Science, 44, 2150-2160, 1997

[19] T.A. Nordheim, L.R. Dartnell, L. Desorgher, A.J. Coates, G.H. Jones, Ionization of the Venusian atmosphere from solar and galactic cosmic rays, Icarus, 245,80-86, 2015

[20] H.S. Porter, C.H. Jackman, A.E.S. Green, Efficiencies for production of atomic nitrogen and oxygen by relativistic proton impact in air, J. Chem. Phys., 65, 154-167, 1976 